\title{
A Flare on AD Leo observed in Optical, UV and Microwaves.
}

\author{
P.D. Byrne, Armagh Observatory, Armagh BT61 906, N Iroland \\ D.E. Gary, Solar Astronomy, Caltoch, Pasadona CA 91125, USA
}

\section{Summary}

\begin{abstract}
We report a simultaneous IUE, VLA and ground-bised photomotric observation of a flare on the Uhe star, ADLeo, on 2ind February 1983. The optical flare was extremely impulsive, lasting in total only shout 3 mins. A relatively longlived $6 \mathrm{~cm}$ flare was observed with the VLA which was initially $100 \%$ polarized. An IUE spectrum, laken $\approx 8 \mathrm{~min}$ after the onset of the optical $U$ band flare, shows more than a factor of 2 increase in the Hell $\lambda 1640 \AA$ emission line. The other mid-transition region lines such as $C I V \lambda \lambda 1548 / 52 \AA$ show almost no response.
\end{abstract}

\section{Introduction}

As part of a coordinated international campaign of inulti-wavelength observation: if the flare star, AD Leo, simultaneous ground-based optical photometry and specroscopy, IUE ultraviolet spectroscopy and VLA microwave observations were made from 2nd to 5 th February 1983 (Byrne et al. 1983, Gary et al. 1988, Byrne et al. 1989). A number of flares were seen independently in the three wavebands but one, on 2nd February 1983 beginning at $\approx 11: 37$ UT, was seen in all three bands simuitaneously. We discuss this flare here.

\section{The optical flare}

The light curve of the optical (U-band) flare is shown in the upper panel of Fig. 1. It Exhibits the classical time-evolution of a simple oplical flare with a fast $(\approx 10 \mathrm{sec})$ rise to peak light, followed by an initially fast $\left(\tau_{1 / 2} \approx 1 \mathrm{sec}\right)$ fall which slows later in the flare. The mis outburst is effertivaly over in $\approx$ 3-4 min. A smaller, secondary flare occurs about 17 min later at $\approx 11: 54 \mathrm{UT}$. 


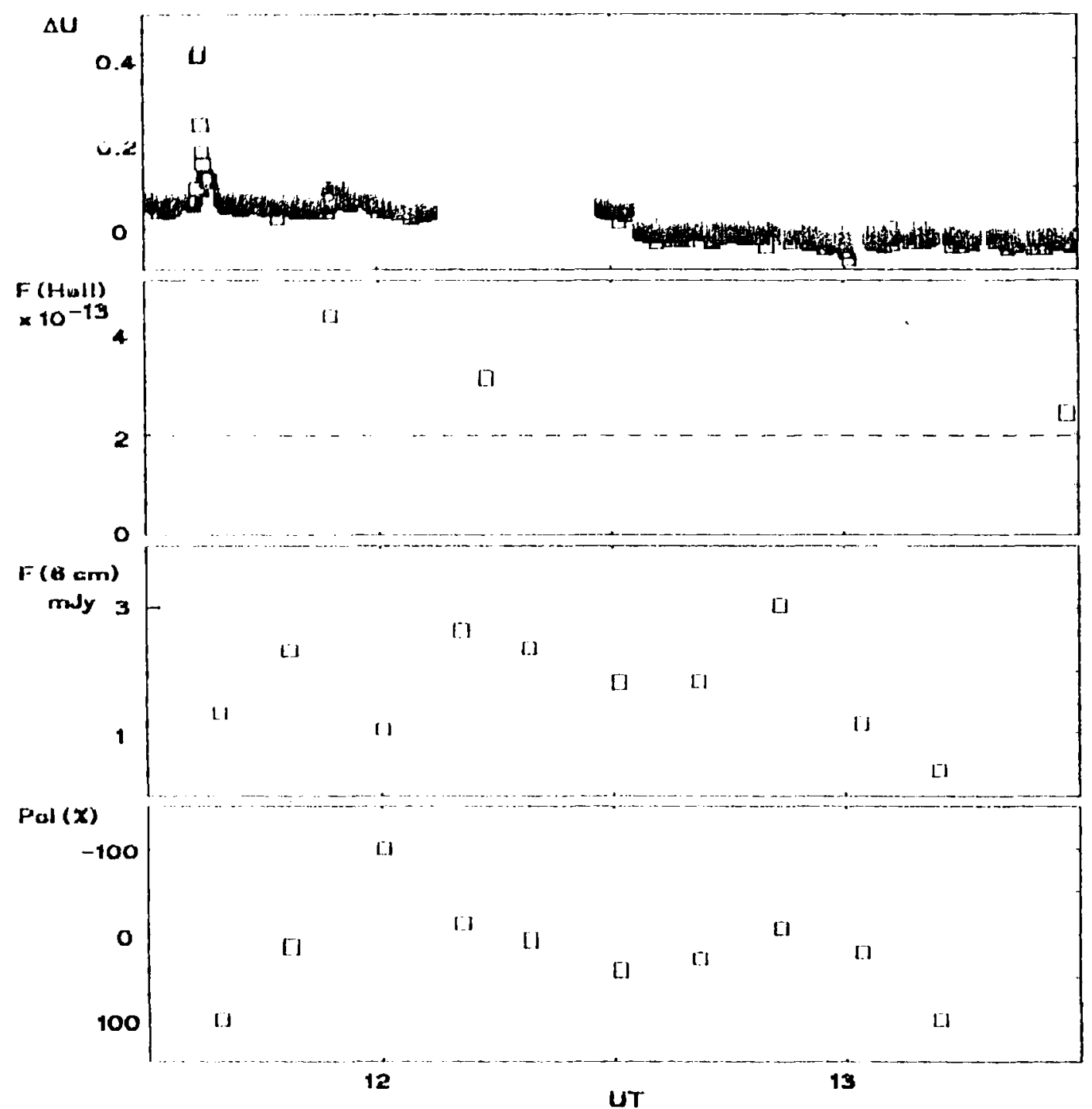

Fig. 1. The flare of 2 Feb 1984 as observed in optical U-band (upper panel), He II $\lambda 1640 \AA$ (second panel), and $6 \mathrm{~cm}$ total flux (thirdpanel) and circular polarization (lower panel). Note that negative polarization is LCP and that the dashed line in the He II plot is the mean preflare level.

\section{The ultraviolet flare}

Seven ultraviolet spectra were taken with the IUE satellite's short wavelength $(1150 \AA-$ $1950 \AA$ ) camera in its low resulution ( $\therefore 5 \AA$ ) mode on 2 February 1983 between 08:00 UT and 14:04 UT. Each of the first six of these was a double exposure wherein the star was moved on the spectrogriph slit between exposures in a direction perpendicular to the dispersion. Each exposure thus obtained was of $20 \mathrm{~min}$ duration. 
Unfoturatelj, no short wavelength exposure was in progress at the time of onset of the optical flare. One was started $\approx 7 \mathrm{~min}$ later, however, and it shows a $\approx 250 \%$ increase in the He $11 \lambda 1640 \AA$ line emission (Fig. 1) but no significant reponse from the other transition region lines (i.e. CII $\lambda 1335 / 6 \AA$ and $C I V \times 1548 / 52 \&$ ). The following spectrum, iaken $20 \mathrm{~min}$ later, shows the He $11 \lambda 1640 \&$ line still enhanced over quiescent. Thu 1 fe response to the flare was also measured: It shows an even more delayed response, peaking in a spectrum centred $\therefore 37 \mathrm{~min}$ after the optical peak and continuing until at least 75 mins after flare peak whun the ultraviolet monitoring ended.

\section{The microwave flare}

$A D$ Leo was also being monitored by the VLA at $6 \mathrm{~cm}$ at the time of the onset of the optical flare. Owing to instrumental difficulties the array was workinus at reduced sensitivity and data recording only began at $\approx 11: 34$ UT. Unfortunately, due to the reduced sensitivity this data must be binned into $10 \mathrm{~min}$ bins to achieve ndequate signal-to-noise. Thius the first measurement of the night includes the flare onset and no measurement of the preflare levil is possible.

Nuvertheless, the $6 \mathrm{~cm}$ data provide interesting observations which are summarized in Fig. 1 as a plot of total intensity and circular polarization against time. The first integration is 100\% left-hand circularly polarized. In the following integration there is no net polus ization while ten minutes later the polarization is again $100 \%$ but in the reverse sense. Significant $6 \mathrm{~cm}$ flare emission is seen over a total of at least $90 \mathrm{~mm}$.

\section{Ciscussion}

The long lasting He $I I$ and Lyce response to the relatively impulsive optical flare ( $1: 1 \mathrm{~h}$ ) is suggestive of a timescale typical of the soft $X$-ray component of solar flares rather than the impulsive hard $X$-rays. In general, the transition region response in solar flares is itself relarively impulsive and this is consistent will, the lack of response from the CIV resonance doublet. It is merefore surprising to find the Hell persisting over such a long time since its temperature of formation in thermal equilibrium is $\log T: 34.7$. A picture consistent with this observation would be one of soft $X$-ray back-heating by the post-flare loop. Evidence for soft $X$-ray photoexcitation of the He atom has been previously *ircussed by Kohl (1977) 
for the solar case and for the late type star - by Byrne and Doyle (1988).

The microwave flare occurs on a similar timescale to the Lya and He II emission. At onset it is completely circularly polarized, however, suggesting a non-thermal process. Even theslower component of the flare cannot easily be interpreted as thermal since a thermal plasmia would produce a flux at Earth ul

$S=2 v^{2} k T R^{2}$

This can be evaluated for a post-flare plasma temperature, $T \approx 10^{7} \mathrm{~K}$ at the distance of $A D I$ eo, i.e. $d: 5 \mathrm{pc}$, and solved for the source radius. The observed flux density of $\approx 2.5 \mathrm{mJy}$ leads to a source radius of $R \cong 75 R$. Filling such a large volume with plasma at temperature $\because 10^{7} \mathrm{~K}$ presents severe difficulties.

\section{References}

Byrne, P.B., Butler, C.J., Andrews, A.D., Rodono, M., Catalano, S., Pazzani, V., Linsky, J.L., Bornman, P., Haisch, B.M.: 1983, Inf. Bull. Var. Stars, No. 2258.

Byrrie, P.B., Doyle, J.G.: 1988, Astron. Astrophys., in press.

Bjirne, P.B., Brown, A., Butler, C.J., Gary, D.E., Linsky, J.L., Simon, T.: 1989, in preparation Gury, D.E., Byrne, P.B., Butler, C.J.: 1988, in Cool Stars, Stellar Systems and the Sun, Eds. J.L. Linsky and R.E. Stencel, Lecture Nalss in Physics, No. 291. Publ. Springer-Verlag, p. 106.

Kohl, J.L.: 1977, Astrophy's. J., 211, 958. 